\title{
PROMOÇÃO À SAÚDE BUCAL DE CRIANÇAS INTERNADAS EM UM HOSPITAL INFANTIL DE ALTA COMPLEXIDADE DE UM MUNICÍPIO DO SUL CATARINENSE
}

\section{Profile of oral health of children in a children's high complexity of hospital of a southern of santa catarina}

Maria Fernanda Gazola1, Luciane Bisognin Ceretta ${ }^{1,2,3}$, Lisiane Tuon ${ }^{1,2}$, Renan Antonio Ceretta ${ }^{3}$, Priscyla Waleska Simões ${ }^{1,2,3}$, Fernanda Guglielmi Faustini Sônego ${ }^{1,3}$

${ }^{1}$ Programa de Residência Multiprofissional em Atenção Básica/Saúde Coletiva Universidade do Extremo Sul Catarinense - UNESC, Criciúma, SC, Brasil.

2 Programa de Pós-Graduação em Saúde Coletiva (Mestrado Profissional) Universidade do Extremo Sul Catarinense - UNESC, Criciúma, SC, Brasil.

${ }^{3}$ Curso de Odontologia - Universidade do Extremo Sul Catarinense - UNESC, Criciúma, SC, Brasil.

\section{Endereço para correspondência:}

\section{Fernanda Guglielmi Faustini Sônego}

Programa de Residência Multiprofissional em Atenção Básica/Saúde Coletiva Universidade do Extremo Sul Catarinense, Av. Universitária, 1105, Bairro Universitário, Criciúma - SC. CEP - 88806-000.

Email: fgfsonego@unesc.net 


\title{
Resumo
}

Objetivo: Investigar as condições de saúde e higiene bucal em crianças internadas em um hospital infantil de alta complexidade localizado num município do Sul Catarinense. Métodos: Estudo transversal, descritivo e de abordagem quantitativa, realizado no Hospital Materno Infantil Santa Catarina, do município de Criciúma-SC, com uma amostra de 80 crianças. Foram coletados dados sociodemográficos das mães das crianças, motivo de internação, aleitamento materno, uso de chupetas ou mamadeira, hábito de adoçar líquidos oferecidos às crianças, dados sobre higiene oral, entre outros. Resultados: A mediana de idade das crianças foi de 18,0 (5,0$36,0)$ meses, de anos de estudo dos responsáveis de 8,8 $( \pm 2,8)$ e da renda familiar de $R \$ 1947,00( \pm 1115,20)$. $56,8 \%$ das mulheres que possuem maior escolaridade trabalham fora, e as que possuem menor escolaridade ficam com os afazeres domésticos e cuidando dos filhos. $70 \%$ dos entrevistados não realizavam a higiene bucal nos internados. 35\% das internações foram provenientes de doenças respiratórias. Conclusão: A população predominante do estudo foi composta por crianças que não mamam no peito, não consomem bebida adoçada, usam mamadeira e chupeta, que não realizaram higiene bucal durante a internação, não apresentaram cárie e alteração na mucosa e foram internadas predominantemente por causa de doenças do aparelho respiratório.

Palavras-chave: Educação em Saúde; Saúde Bucal; Odontopediatria; Saúde Coletiva; Epidemiologia Descritiva.

\begin{abstract}
Objective: To investigate health and oral hygiene in children admitted to a children's hospital highly complex located in a city in South of Santa Catarina. Methods: Crosssectional, descriptive study with a quantitative approach, performed at the Hospital Materno Infantil Santa Catarina of Criciúma-SC municipality, with a sample of 80 children. We collected demographic data of the mothers of children, reason for hospitalization, breastfeeding, use of pacifiers or bottle, liquid sweeten usual children available, data on oral hygiene, among others. Results: The median age was 18.0 (5.0 to 36.0) months, years of study of those responsible of $8.8( \pm 2.8)$, and family income of R \$ 1947.00 (1115.20 \pm ). 56.8\% of women who have higher education
\end{abstract}


work outside and those with less education are left with the household chores and taking care of the children, $70 \%$ of respondents did not perform oral hygiene in hospitalized. 35\% of admissions were from respiratory diseases. Conclusion: The predominant study population consisted of children who are not breastfed, do not consume sweetened drink, use bottles and pacifiers, who did not perform oral hygiene during hospitalization, did not show decay and changes in the mucosa, and admitted predominantly by respiratory diseases.

Keywords: Health Education; Oral Health; Pediatric Dentistry; Public Health; Descriptive Epidemiology.

\section{INTRODUÇÃO}

Na evolução do processo saúde-doença, a Odontologia tem se demonstrado puramente curativa, em detrimento da promoção e prevenção de saúde, de modo a não atingir os objetivos sociais. Embora a Constituição brasileira afirme a "saúde como direito de todos e dever do Estado", sendo a universalidade do acesso às ações e aos serviços de saúde para a sua promoção, proteção e recuperação um dos princípios constitucionais do sistema de saúde do país, isso vem se concretizando pouco no que diz respeito aos serviços odontológicos ${ }^{1}$.

Mesmo com os progressos na área odontológica, ainda falta consciência da importância das avaliações odontológicas em pacientes pediátricos internados para que possam colaborar para o bem-estar dos mesmos². Sabe-se que algumas alterações bucais também podem interferir na saúde geral, necessitando as crianças hospitalizadas receberem cuidados e orientações específicas para as diferentes situações. Crianças hospitalizadas apresentam debilidade sistêmica e, portanto, maior vulnerabilidade ao desenvolvimento destas doenças, onde seus efeitos não são limitados, podendo levar a quadros infecciosos que resultam em comprometimento do quadro sistêmico ${ }^{3}$.

Nesse contexto, a prevalência de cárie dentária no Brasil tem diminuído nos últimos anos. A Pesquisa Nacional de Saúde Bucal ${ }^{4}$, realizada em 2010, em 177 municípios, com 38 mil pessoas de diferentes grupos etários, revela uma redução de $26 \%$ da doença cárie nas crianças de 12 anos desde 2003. Porém, em piores 
Artigo Original

Atenção à Saúde

condições socioeconômicas observa-se ainda uma alta prevalência de cárie, explicada pelas más condições de vida as quais a população é submetida, assim como também pela falta de informação 4 .

Dentre os fatores socioeconômicos, o baixo poder aquisitivo restringe a aquisição de bens e serviços, além das necessidades básicas não serem muitas vezes supridas, debilitando assim a saúde geral. Por outro lado, as infecções bucais afetam a dieta e o estado nutricional uma vez que causam impacto na capacidade de alimentar-se. A dieta tem consequência direta com a integridade da cavidade bucal, como também, na progressão de doenças bucais e sistêmicas, tornando-se um ciclo vicioso ${ }^{5}$.

As más condições bucais podem atuar como fatores de risco e foco de disseminação de microrganismos, com efeito metastático sistêmico, especialmente em pessoas que apresentam imunidade baixa ${ }^{6,7}$. A microbiota bucal sofre influência de fatores externos, dentre eles, antibioticoterapia ou corticoterapia, permanência em ambientes hospitalares, estado nutricional, higiene bucal e fatores intrínsecos ao paciente, pela possibilidade de alterar a imunidade local e a sistêmica e por selecionar espécies bacterianas ${ }^{8}$. Assim, o tratamento odontológico em pacientes hospitalizados contribui para a prevenção e/ou melhora da condição sistêmica do mesmo.

No ambiente hospitalar a inserção do cirurgião-dentista tem sido discreta ao longo das últimas décadas. A importância da Odontologia neste novo cenário de atuação pode significar um grande avanço da saúde, pois a diminuição dos focos bucais de infecção poderia resultar num melhor restabelecimento do paciente, assim minimizando possíveis quadros de dor no período de internação.

Mediante o exposto, o presente estudo buscou investigar condições de saúde e higiene bucal em crianças internadas em um hospital infantil de alta complexidade localizado num município do Sul Catarinense.

\section{MÉTODOS}

Foi realizado um estudo transversal, descritivo e de abordagem quantitativa, desenvolvido no Hospital Materno Infantil Santa Catarina, no município de CriciúmaSC, no período de setembro a dezembro de 2013. A pesquisa foi iniciada após 
aprovação pelo Comitê de Ética e Pesquisa em Seres Humanos da Universidade do Extremo Sul Catarinense, sob os pareceres 372.487/2013 e 421.870/2013 e autorização do local onde foi realizada a pesquisa.

A população estudada foi composta por 80 pacientes infantis hospitalizados, com idade de 0 a 10 anos. Participaram do estudo os indivíduos em que os pais e/ou responsáveis autorizaram a pesquisa assinando o Termo de Consentimento Livre e Esclarecido, que estavam internados, independentemente da idade, crianças que estavam em condições de saúde que viabilizassem a realização do estudo e que estivessem acompanhadas de seus cuidadores e/ou responsáveis. Foram excluídas as crianças com doenças infectocontagiosas que inviabilizassem o contato com o paciente.

Os pais e/ou responsáveis foram abordados nos leitos hospitalares, sendo apresentado um formulário com perguntas sobre a idade da criança, sexo, anos de estudo da mãe, se a mãe trabalha fora, renda da família, tipo de moradia, motivo e tempo da hospitalização, se as crianças mamam no peito, se as mães costumam adoçar as bebidas dos filhos, o uso de mamadeira e chupeta, a realização da higiene bucal durante o internamento, como foi realizada, quando e quem faz, presença de dor de dente, presença de cárie e/ou alteração de mucosa.

Posteriormente, foram realizadas algumas orientações de higiene, escovação, alimentação, importância da saúde bucal e sua relação com a saúde geral para os pais e/ou responsáveis e para os internados. Em seguida, uma avaliação da cavidade bucal foi feita nos internados, sendo solicitado que a criança abrisse a boca para observação de alterações de mucosa e lesões cariosas. $\mathrm{Na}$ presença de alteração, os pais e/ou responsáveis foram aconselhados a levar a criança a uma Unidade de Saúde mais próxima que tivesse um cirurgião-dentista ou mesmo a um consultório particular.

Após a coleta de dados, foi elaborado um banco de dados e realizada a análise estatística no software Statistical Package for the Social Sciences (SPSS) versão 20.0. Foram calculadas algumas medidas descritivas, como média e desvio padrão ou mediana e intervalo interquartil para as variáveis quantitativas e frequência absoluta e relativa para as qualitativas.

A investigação da existência de associação entre as variáveis qualitativas, como sexo e escolaridade, foi realizada através da aplicação do teste qui-quadrado 
Artigo Original

Atenção à Saúde

de associação ou independência, e nas tabelas de contingência utilizou-se a correção de Yates. Todos os cálculos analíticos foram realizados com um nível de significância $\alpha=0,05$ e intervalo de confiança de $95 \%$.

\section{RESULTADOS}

A pesquisa contou com 80 entrevistados, sendo que a mediana de idade das crianças foi de 18,0 (5,0-36,0) meses, a média de anos de estudo dos responsáveis foi de $8,8( \pm 2,8)$ anos e a média da renda familiar foi de $R \$ 1947,00( \pm R \$ 1115,20)$.

A Tabela 1 mostra características da amostra, estratificadas segundo a escolaridade das mães.

Tabela 1. Características da amostra, estratificadas por anos de estudo da mãe.

\section{Anos de estudo}

\begin{tabular}{|c|c|c|c|c|}
\hline Variável & Total & $\begin{array}{c}1 \text { a } 9 \text { anos } \\
n(\%) \\
n=43\end{array}$ & $\begin{array}{c}10 \text { ou mais } \\
n(\%) \\
n=37\end{array}$ & $\mathbf{p}$ \\
\hline \multicolumn{5}{|l|}{ Sexo } \\
\hline Feminino & 40 & $25(62,5)$ & $15(37,5)$ & \multirow[t]{2}{*}{0,178} \\
\hline Masculino & 40 & $18(45,0)$ & $22(55,0)$ & \\
\hline \multicolumn{5}{|c|}{ Mãe Trabalha Fora } \\
\hline Sim & 44 & $19(43,2)$ & $25(56,8)$ & \multirow[t]{2}{*}{0,061} \\
\hline Não & 36 & $24(66,7)$ & $12(33,3)$ & \\
\hline \multicolumn{5}{|c|}{ Tipo de residência } \\
\hline Alvenaria & 52 & $26(50,0)$ & $26(50,0)$ & \multirow[t]{3}{*}{0,388} \\
\hline Madeira & 11 & $8(72,7)$ & $3(27,3)$ & \\
\hline Mista & 17 & $9(52,9)$ & $8(47,1)$ & \\
\hline \multicolumn{5}{|l|}{ Propriedade } \\
\hline Própria & 23 & $11(47,8)$ & $12(52,2)$ & \multirow[t]{2}{*}{0,669} \\
\hline Alugada & 57 & $32(56,1)$ & $25(43,9)$ & \\
\hline \multicolumn{5}{|l|}{ Mama no peito } \\
\hline Sim & 15 & $10(66,7)$ & $5(33,3)$ & \multirow[t]{2}{*}{0,409} \\
\hline Não & 67 & $33(50,8)$ & $34(49,2)$ & \\
\hline \multicolumn{5}{|c|}{ Adoça a bebida } \\
\hline Sim & 26 & $17(65,4)$ & $9(34,6)$ & \multirow[t]{2}{*}{0,227} \\
\hline Não & 54 & $26(48,1)$ & $28(51,9)$ & \\
\hline \multicolumn{5}{|c|}{ Usa mamadeira } \\
\hline Sim & 49 & $28(57,1)$ & $21(42,9)$ & \multirow[t]{2}{*}{0,593} \\
\hline Não & 31 & $15(48,4)$ & $16(51,6)$ & \\
\hline \multicolumn{5}{|l|}{ Usa chupeta } \\
\hline Sim & 43 & $24(55,8)$ & $19(44,2)$ & \multirow[t]{2}{*}{0,862} \\
\hline Não & 37 & $19(51,4)$ & $18(48,6)$ & \\
\hline
\end{tabular}

Fonte: Próprio Autor 
Evidencia-se neste estudo que as mulheres com maior escolaridade trabalham fora $(n=25 ; 56,8 \%)$ e as que possuem menor escolaridade ficam com os afazeres domésticos e cuidando dos filhos ( $n=24 ; 66,7 \%$ ). No entanto, tal associação foi estatisticamente limítrofe $(p=0,061)$.

Sobre o tipo de residência, morar em casa de alvenaria não revelou associação entre possuir entre 1 a 9 anos de estudo, como entre 10 anos ou mais ( $n=26 ; 50,0 \%$ ). Para a casa mista observou-se que a maioria dos indivíduos que moram em casa mista apresentaram entre 1 a 9 anos de estudo ( $n=9 ; 52,9 \%$ ). Em relação à casa de madeira os achados foram similares. Com relação à amamentação, o estudo mostra que mães que estudam menos amamentam mais os filhos ( $n=10 ; 66,7 \%)$.

Evidencia-se que as mães que possuem menor escolaridade utilizam mais a mamadeira como recurso para alimentar os seus filhos $(n=28 ; 57,1 \%)$ e as que não a utilizam apresentaram os mesmos resultados.

A Tabela 1 ilustra ainda que a utilização de chupeta para acalmar os filhos independe do nível de escolaridade da mãe, sendo que aproximadamente $50 \%$ a utiliza em ambos os grupos (de menor e de maior escolaridade) $(p=0,862)$.

Em relação ao motivo da hospitalização das crianças, a doença do aparelho respiratório foi encontrada em 28 (35,0\%) internações, seguindo-se da doença do aparelho digestivo em 11 (14,0\%) internações e doenças do sangue e dos órgãos hematopoiéticos e alguns transtornos imunitários ( $n=11 ; 13,8 \%)$, conforme apresenta a Tabela 2.

Tabela 2. Motivo da hospitalização.

\begin{tabular}{lc}
\hline Motivo da hospitalização & $\begin{array}{c}\mathbf{n}(\%) \\
\mathbf{n = 8 0}\end{array}$ \\
\hline $\begin{array}{l}\text { Doença do aparelho respiratório } \\
\text { Doença do aparelho digestivo }\end{array}$ & $28(35,0)$ \\
Doenças do sangue e dos órgãos hematopoiéticos e & $11(14,0)$ \\
alguns transtornos imunitários & $11(14,0)$ \\
Doenças do aparelho geniturinário & $9(11,0)$ \\
Doenças cardíacas & $7(8,8)$ \\
Sintomas, sinais e achados anormais em exames & $3(3,8)$ \\
clínicos e de laboratório, não classificados em outra & \\
parte & $3(3,8)$ \\
Doenças dos olhos e anexos & $2(2,5)$ \\
Doenças de pele e tecido subcutâneo & \\
\hline \hline
\end{tabular}




\begin{tabular}{|c|c|}
\hline Motivo da hospitalização & $\begin{array}{l}\mathrm{n}(\%) \\
\mathrm{n}=80\end{array}$ \\
\hline Gravidez, parto e puerpério & $2(2,5)$ \\
\hline $\begin{array}{l}\text { Lesões, envenenamentos e algumas consequências } \\
\text { externas }\end{array}$ & $1(1,3)$ \\
\hline Doenças do sistema nervoso & $1(1,3)$ \\
\hline Doenças do ouvido & $1(1,3)$ \\
\hline Transtornos mentais e comportamentais & $1(1,3)$ \\
\hline
\end{tabular}

\section{Fonte: Próprio Autor}

Analisando o uso de medicamentos, 60 (75,0\%) crianças fizeram uso apenas no hospital. Quando questionadas sobre a amamentação, 65 (81,3\%) relataram não amamentar e apenas $15(18,8 \%)$ continuam amamentando. Sobre adoçar a bebida das crianças, 54 (67,5\%) mencionaram não adoçar a bebida, enquanto $26(32,5 \%)$ afirmaram colocar açúcar na bebida das crianças. No que diz respeito ao uso da mamadeira, $49(61,3 \%)$ relataram utilizá-la. E sobre o costume das crianças de usar chupeta, tal questão foi confirmada por $43(52,8 \%)$ dos responsáveis.

Quanto à higiene bucal durante o internamento da criança, $56(70,0 \%)$ dos responsáveis relataram que não realizaram tal procedimento e 24 (30,0\%) afirmaram fazer a higiene bucal dos internados. Dos responsáveis que realizaram a higiene durante o internamento, $20(25,0 \%)$ utilizaram escova e creme dental. Das que higienizavam, quando questionadas quantas vezes realizavam a higiene bucal, verificou-se que $12(15,0 \%)$ crianças realizavam a higiene duas vezes ao dia, 8 $(10,0 \%)$ três vezes ao dia e $4(5,0 \%)$ uma vez ao dia. Sobre quem higienizava a cavidade bucal, $23(28,8 \%)$ eram realizadas pelo acompanhante.

Em relação à higiene bucal durante o internamento, na Tabela 3 pode-se observar que as mães com maior escolaridade realizaram a higiene bucal durante a internação ( $n=15 ; 62,5 \%)$, e dentre as que não realizaram a higiene bucal no internamento, a maioria apresentou menor escolaridade $(n=34 ; 60,7 \%)$. 
Tabela 3. Associação entre higiene bucal durante o internamento, forma de higiene, cárie e alteração de mucosa e os anos de estudo da mãe.

\begin{tabular}{|c|c|c|c|c|}
\hline \multirow{2}{*}{ Variável } & \multirow[b]{2}{*}{ TOTAL } & \multicolumn{2}{|c|}{ Anos de estudo } & \multirow[b]{2}{*}{$\mathbf{p}$} \\
\hline & & $\begin{array}{c}1 \text { a } 9 \text { anos } \\
n(\%) \\
n=43\end{array}$ & $\begin{array}{c}10 \text { ou mais } \\
n(\%) \\
n=37\end{array}$ & \\
\hline \multicolumn{5}{|c|}{$\begin{array}{l}\text { Higiene bucal durante } \\
\text { internamento }\end{array}$} \\
\hline Sim & 24 & $9(37,5)$ & $15(62,5)$ & 0,096 \\
\hline Não & 56 & $34(60,7)$ & $22(39,3)$ & \\
\hline \multicolumn{5}{|l|}{ Qual forma de higiene } \\
\hline Não higienizava & 56 & $34(60,7)$ & $22(39,3)$ & 0,233 \\
\hline Creme dental e escova & 20 & $8(40,0)$ & $12(60,0)$ & \\
\hline Gaze & 3 & $1(33,3)$ & $2(66,7)$ & \\
\hline Fralda & 1 & $0(0,0)$ & $1(100,0)$ & \\
\hline \multicolumn{5}{|l|}{ Cárie } \\
\hline Não houve avaliação & 1 & $1(100,0)$ & $0(0,0)$ & 0,115 \\
\hline Sim & 12 & $3(27,3)$ & $8(72,7)$ & \\
\hline Não & 68 & $39(57,4)$ & $29(42,6)$ & \\
\hline \multicolumn{5}{|l|}{ Alteração de mucosa } \\
\hline Não houve avaliação & 1 & $1(100,0)$ & $0(0,0)$ & 0,510 \\
\hline Sim & 6 & $4(66,7)$ & $2(33,3)$ & \\
\hline Não & 73 & $38(52,1)$ & $35(47,9)$ & \\
\hline
\end{tabular}

Quanto à dor, nenhuma criança relatou sentir dor de dente durante a internação, por isso não houve encaminhamentos escritos. Das crianças investigadas, $68(85,0 \%)$ não apresentaram cárie. Sobre a alteração da mucosa bucal, a mesma foi encontrada em $6(7,5 \%)$ pacientes, os quais estavam internados devido a estomatite, por isso não foram encaminhados ao cirurgião-dentista.

\section{DISCUSSÃO}

Nosso estudo revelou mediana de idade dos internados de 18,0 meses, porém a literatura aponta que o maior número de internações é de crianças de $0 \mathrm{a}$ 12 meses, como mostra uma pesquisa sobre as internações de crianças de 0 a 9 anos de idade residentes no município de São Paulo ${ }^{9}$. A média de internações pediátricas no Brasil no período de 2002 a 2006 foi de 207,01 internações por 1.000 crianças de 0 a 12 meses e 64,97 por 1000 crianças com idade de 12 a 48 meses $^{9}$. 
Sobre a associação da mãe trabalhar fora aos anos de estudo, os dados do presente estudo sugerem que mães que estudaram 10 anos ou mais trabalham fora, corroborando com alguns estudos, que relatam que a proporção de mulheres trabalhando cresce em todos os níveis de escolaridade ${ }^{10}$. Nesse sentido, a escolaridade dos pais é importante, principalmente a materna, o que determina os cuidados que a criança recebe: a percepção dos pais sobre a criança, o modo de cuidar da criança doente, a capacidade dos pais de buscar auxílio médico, o atendimento médico recebido e a adesão às recomendações ${ }^{9}$.

Sobre a amamentação, é interessante observar que cerca de $67 \%$ das mães que tem até 9 anos de estudo amamentam, em contraposição a aproximadamente $33 \%$ das que tem maior nível de escolaridade, característica essa que difere de um estudo transversal realizado num hospital público de São Paulo ${ }^{11}$ sobre aleitamento materno e condições socioeconômicas, sugerindo que quanto maior a escolaridade da mãe, maior o tempo de amamentação. Em nosso estudo, o uso da mamadeira ocorreu em $61,3 \%$ das crianças, e 53,8\% utilizam a chupeta; assim, introdução da chupeta no enxoval do bebê e sua aquisição antes do nascimento denotam um aspecto prático e utilitário da chupeta para a mãe, caso ela necessite utilizá-la, no intuito de acalmar o seu filho. Por isso, a chupeta é importante para a mãe ${ }^{11}$.

No presente estudo, o maior motivo de internação das crianças deu-se pelas doenças do trato respiratório. Tais achados corroboram com outros estudos que apontam a doença respiratória como uma das principais causas de internação hospitalar. A principal causa de internação hospitalar na faixa etária de 0 a 5 anos, mediante dados do Sistema de Informações Hospitalares do Sistema Único de Saúde, no período de 2002 a 2006, em São Paulo e no Brasil, ocorreu pelas doenças do trato respiratório, correspondendo a $32,5 \%$ e $38,4 \%$, respectivamente, do total de internações ${ }^{9}$.

A manutenção da saúde bucal depende não somente do dentista, mas também da participação ativa do paciente. Orientações sobre higiene bucal devem ser dadas individualmente ou em grupos, com a participação dos familiares. A ação preventiva e educacional deve ser realizada com cada paciente. O paciente deve ser informado de que, mesmo na presença de sangramentos, a higiene bucal deve ser mantida, visando a preservação da integridade periodontal ${ }^{12}$. Associada a medidas educativas e preventivas, ações de recuperação do dano já estabelecido, a 


\section{Artigo Original}

\section{Atenção à Saúde}

adequação do meio bucal tem sido uma alternativa proposta para a atenção odontológica em ambiente hospitalar ${ }^{13}$. A promoção da saúde, em ambiente hospitalar, compreende ações para aumento da resistência do hospedeiro, redução de focos de infecções bucais para que o restabelecimento do paciente se dê o mais rápido possível.

Uma pesquisa qualitativa evidenciou que os participantes - equipe de saúde e usuários da Unidade de Internação Pediátrica do Hospital Universitário da Universidade Federal de Santa Catarina - percebem como muito importante, e até fundamental, a participação efetiva do cirurgião-dentista na atenção à saúde da criança hospitalizada. Essa percepção tem origem em experiências vividas com a própria atenção odontológica e também por crenças e conhecimentos acerca do que é idealizado para a prática do cirurgião-dentista na atenção integral à saúde da criança ${ }^{14}$.

Um outro estudo sugere que atividades educativo-preventivas, além de auxiliarem na prevenção de infecções hospitalares e contribuírem para o bem-estar e qualidade de vida do indivíduo, representam também uma escolha barata (nível primário de prevenção), simples, viável e de extrema importância e necessidade ${ }^{15}$. A promoção da saúde bucal em ambiente hospitalar proporciona conhecimento, motiva os pacientes e seus acompanhantes a aquisição de bons hábitos e visa a assistência integral e mais humanizada do paciente hospitalizado. Tais ações são plausíveis na introdução da higiene bucal dos pacientes à rotina hospitalar, reduzindo o biofilme dentário e, consequentemente, o risco de infecções provenientes da microbiota bucal ${ }^{15}$.

O cirurgião-dentista poderá ter um papel significativo dentro de um hospital, tornando-se membro de equipe profissional, o que permitirá um acesso mais global dos pacientes, promovendo consultas de urgência para bebês, crianças, adolescentes, adultos e pessoas com necessidades especiais de saúde, além das oportunidades educativas. O profissional, em parceria com colegas médicos, poderá desenvolver a educação em saúde continuada ${ }^{16}$.

Conclui-se com nosso estudo que a população predominante do estudo foi composta por crianças que não mamam no peito, não consomem bebida adoçada, usam mamadeira e chupeta, que não realizaram higiene bucal durante a internação, não apresentaram cárie e alteração na mucosa e foram internadas 
Artigo Original

Atenção à Saúde

predominantemente por doenças do aparelho respiratório.

\section{REFERÊNCIAS}

1. Narvai PC, Frazão P, Roncalli AG, Antunes JLF. Cárie dentária no Brasil: declínio, polarização, iniqüidade e exclusão social. Rev panam salud pública. 2006;19(6):385-93.

2. Chapper A, Goldani MZ. A participação de odontólogos em equipes multidisciplinares. Rev Fac Odontol Porto Alegre. 2004;45(2):3-5.

3. Amaral KC, Tenório MDH, Dantas AB. Condição de saúde bucal de crianças internas em hospitais da cidade de Maceió-AL. Odontol clín-cient. 2007;5(4):267-73.

4. Brasil. Projeto SBBrasil 2010 - Pesquisa Nacional de Saúde Bucal. Brasília: Ministério da Saúde; 2010.

5. Touger-Decker R, Mobley CC. Position of the American Dietetic Association: oral health and nutrition. Chicago: J Am Diet Assoc; 2007. p. 1418-28.

6. Lindhe J, Karring T, Lang N. Tratado de Periodontia Clínica e Implantologia. 4 ed. Rio de Janeiro: Guanabara Koogan; 2005.

7. Williams RC, Offenbacher S. Medicina Periodontal. São Paulo: Série Periodontologia 2000; 2005.

8. Morais TMN, Silva A, Avi A, Souza PHR, Knobel E, Camargo LFA. A importância da atuação odontológica em pacientes internados em unidade de terapia intensiva. Rev Bras Ter Intensiva. 2006;18(4):412-7.

9. Ferrer APS. Estudo das causas de internação hospitalar das crianças de 0 a 9 anos de idade no município de São Paulo. São Paulo: Universidade de São Paulo; 2009.

10. Itaboraí NR. Trabalho feminino e mudanças na família no Brasil (1984-1996): comparações por classe social. Ouro Preto, Minas Gerais: XIII Encontro da Associação Brasileira de Estudos Populacionais; 2002.

11. Escobar AMU, Ogawa AR, Hiratsuka M, Kawashita MY, Teruya PY, Tomikawa SO, et al. Aleitamento materno e condições socioeconômico-culturais: fatores que levam ao desmame precoce. Rev bras saúde matern infant. 2002;2(3):253-61.

12. Brasil. Manual de atendimento da criança com desnutrição grave em nível hospitalar. Brasília: Ministério da Saúde; 2005.

13. Gomes AC, Biella VDA, Mastrantonio SDS, Neves LT. O tratamento restaurador atraumático (TRA) como alternativa de tratamento em bebês portadores de fissura: Relato de caso clínico. Rev odontol Araçatuba. 2003;24(2):52-5. 
14. Mattevi GS. A Participação do cirurgião-dentista na atenção à criança no contexto hospitalar: percepções de usuários e equipe de saúde do Hospital Universitário da Universidade Federal de Santa Catarina. Florianopolis: Universidade Federal de Santa Catarina; 2010.

15. Euzébio LF, Viana KA, Oliveira Cortines AA, Costa LR. Atuação do residente cirurgião-dentista em equipe multiprofissional de atenção hospitalar à saúde materno-infantil. ROBRAC. 2013;22(60).

16. Aranega AM, Bassi APF, Ponzoni D, Wayama MT, Esteves JC, Garcia Junior IR. Qual a importância da Odontologia Hospitalar? Rev bras odontol. 2012;69(1):903. 